\section{Human pluripotent stem cell-derived organoids and their potential to reduce the use of animal models in research}

\author{
Salvatore Simmini, ${ }^{1}$ Allen C. Eaves, ${ }^{2,3}$ \\ Sharon A. Louis, ${ }^{2}$ Wing Chang1 \\ 1STEMCELL Technologies UK Ltd., \\ Cambridge, UK; 2STEMCELL \\ Technologies Inc., Vancouver BC, \\ Canada; ${ }^{3}$ Terry Fox Laboratory, BC \\ Cancer, Vancouver, BC, Canada
}

\begin{abstract}
Efficient and reproducible generation of tissue-specific organoids from Human Pluripotent Stem Cells (hPSCs) represents one of the key tools for reducing the use of animals in research. STEMCELL Technologies is committed to optimizing workflows that efficiently support the generation and maintenance of multiple types of organoid cultures derived from hPSCs.
\end{abstract}

\section{Introduction}

Organoids are advanced three-dimensional culture systems that exhibit a high degree of self-organization and multi-lineage differentiation, thus they recapitulate many of the characteristics of their tissue of origin. Recent optimization of in vitro culture conditions and protocols allows for a re-creation of the multistep in vivo cell fate decision pathways that support generating gastric and intestinal organoids from Human Pluripotent Stem Cells (hPSCs). ${ }^{1-3}$ Organoids derived from hPSCs represent an attractive tool for drug screening, disease modeling, and cell replacement therapy. We have developed STEMdiff ${ }^{\text {TM }}$ Gastric Organoid Differentiation Kit and STEMdiff ${ }^{\text {TM }}$ Intestinal Organoid Kit, both of which include specialized serum-free media that promote differentiation of hPSCs through the developmental stages of definitive endoderm, foregut and $\mathrm{mid} /$ hindgut, and then to gastric and intestinal organoids, respectively. Here we show that gastrointestinal organoids derived from hPSCs have a variety of tissue-specific characteristics that make them attractive alternatives to the use of animal models in research and in drug discovery.

\section{Materials and Methods \\ STEMdiff ${ }^{\mathrm{TM}}$ Gastric Organoid Kit}

This kit contains 2 basal media and 4 supplements, which are combined to prepare complete media used in the 5 stages of gastric organoid formation. hPSCs maintained in $\mathrm{mTeSR}^{\mathrm{TM}} 1$ were dissociated into clumps and cultured in Endoderm Medium (days 1 - 3, Stage 1). The resulting monolayers were then transferred to Posterior Foregut Medium (days 4 - 7, Stage 2) that generate gastric spheroid progenitor cells. These spheroids are then seeded in Corning ${ }^{\circledR}$ Matrigel ${ }^{\circledR}$ and cultured in Gastric Organoid Growth Medium (days 7 26, Stage 3/4) to generate gastric organoids. These organoids can be fully differentiated in Gastric Organoid Medium, or mechanically dissociated, then subsequently passaged and cultured in Gastric Expansion Medium for extended periods of time ( $\geq$ P10, Gastric Organoid Expansion). Immunofluorescence and gene expression analysis of gastric organoids was performed both at the end of differentiation ( $\geq$ day 34 ) and during organoid expansion (P3; >3 organoids per analysis; $\mathrm{n}=2$ Human Embryonic Stem Cell [hESC] lines and 3 Induced Pluripotent Stem Cell [iPSC] lines).

\section{STEMdiff ${ }^{\mathrm{TM}}$ Intestinal Organoid Kit}

This kit contains 2 basal media and 3 supplements, which are combined to prepare complete media used in the 3 stages of intestinal organoid formation. hPSCs maintained in $\mathrm{mTeSR}^{\mathrm{TM}} 1$ are dissociated into clumps and cultured in Endoderm Medium (days 1 - 3, Stage 1). The resulting mono-
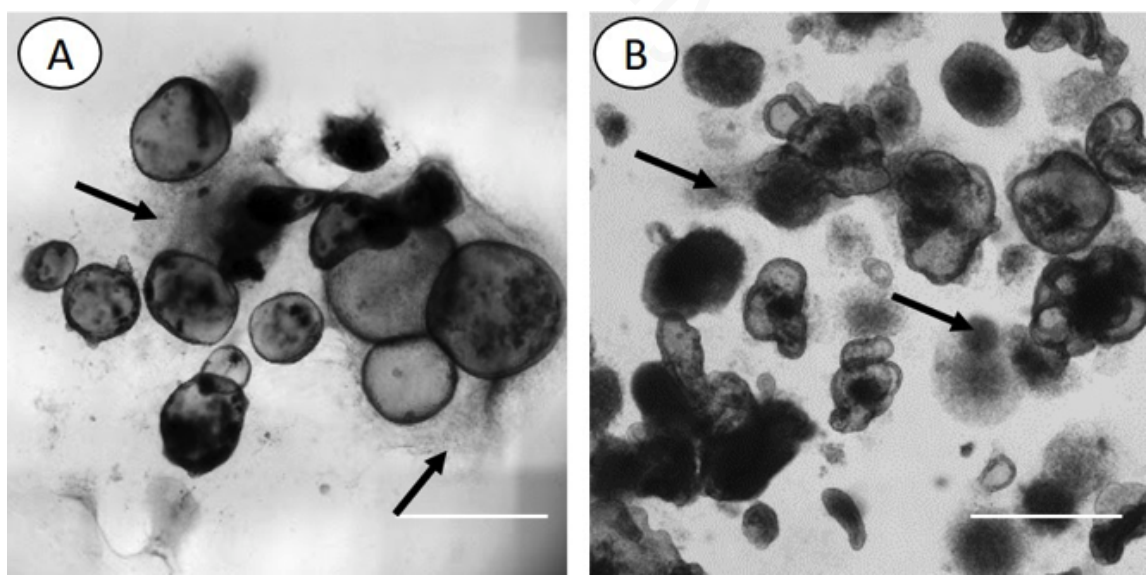

Correspondence:Salvatore Simmini, STEMCELL Technologies UK Ltd., Cambridge, UK.

E-mail: salvatore.simmini@stemcell.com

Key words: Human pluripotent stem cells; organoids; intestine; stomach.

Disclosures: SS and WC are employees of STEMCELL Technologies Ltd., Cambridge (UK) SL, AE are employees of STEMCELL Technologies Inc., Vancouver (Canada).

Conference presentation: This paper was presented at the Third Centro 3R Annual Meeting - L'era delle 3R: modelli in silico, in vitro e in vivo per promuovere la ricerca traslazionale 30 September - 1 October 2021, Evento online organizzato dal Politecnico di Torino.

Received for publication: 9 July 2021.

Accepted for publication: 7 September 2021.

This work is licensed under a Creative Commons Attribution NonCommercial 4.0 License (CC BY-NC 4.0).

(c) Copyright: the Author(s), 2021

Licensee PAGEPress, Italy

Biomedical Science and Engineering 2021; 4(s1):138 doi:10.4081/bse.2021.138

layers are then transferred to Mid/Hindgut Medium (days 4 - 9, Stage 2) that generated intestinal spheroid progenitor cells. Next, these spheroids are seeded in Corning $®$ Matrigel ${ }^{\circledR}$ and expanded in Intestinal Organoid Growth Medium $(\geq \mathrm{P} 10)$ to generate intestinal organoids. Immunofluorescence and gene expression analysis of intestinal organoids was performed during organoid expansion $(\mathrm{P} 3 ;>3$ organoids per analysis; $\mathrm{n}=2 \mathrm{hESC}$ and 2 iPSC lines).

Figure 1. Morphology of gastric (A) and intestinal (B) organoid cultures derived from hPSCs. Organoids in both cultures are surrounded by supportive mesenchymal cells observed as flat, web-like cells (arrows). Scale bars $=1 \mathrm{~mm}$. 


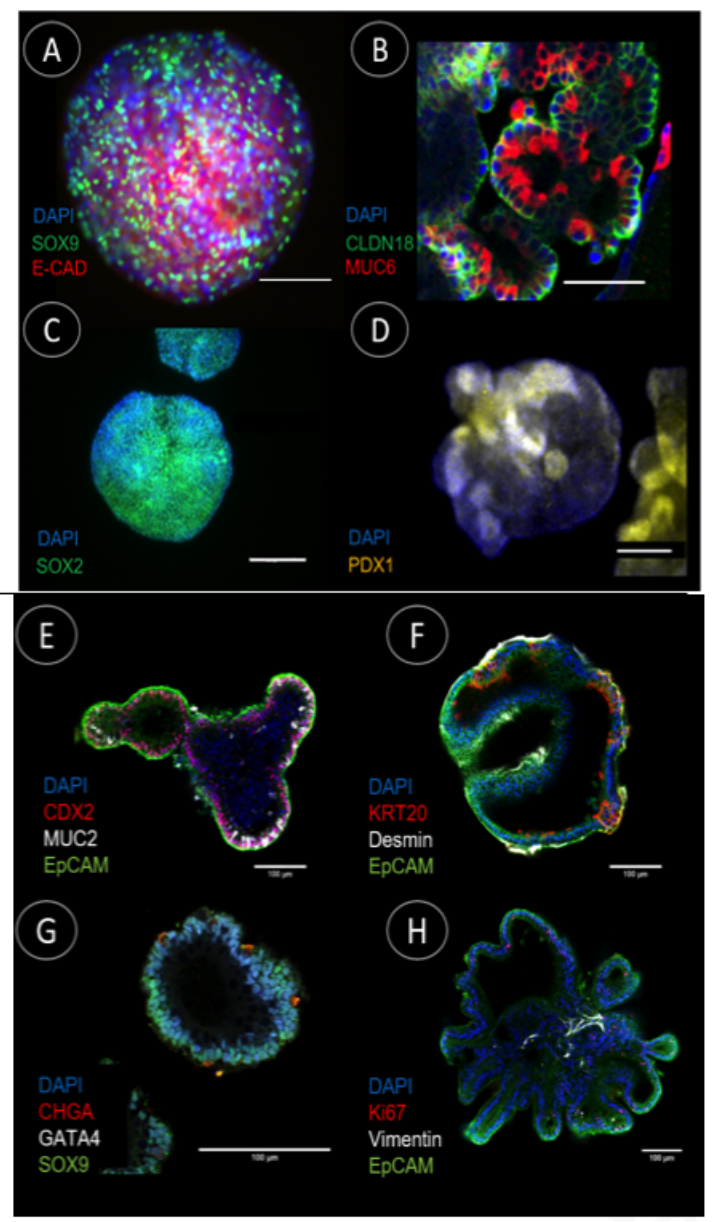

Figure 2. Gastric and intestinal organoids derived from hPSCs express tissue-specific markers. Gastric organoids express gastric markers such as SOX9, E-CAD (A), CLDN18, MUC6 (B), SOX2 (C), and PDX1 (D). Intestinal organoids demonstrate robust expression of intestinal markers such as CDX2, MUC2, EpCAM (E), KRT20, desmin (F), CHGA, GATA4, SOX9 (G), Ki67, and vimentin (H). Scale bars $=100 \mu \mathrm{m}$.

\section{Results}

STEMdiff ${ }^{\mathrm{TM}}$ Gastric Organoid Differentiation Kit and STEMdiff ${ }^{\mathrm{TM}}$ Intestinal Organoid Kit support the generation of organoids that are composed of a polarized epithelium surrounded by mesenchymal cells (Figure 1 and Figures 2A, $2 \mathrm{~B}, 2 \mathrm{E}, 2 \mathrm{~F}$, and $2 \mathrm{H})$.
Gastric organoids are composed of progenitor cells (SOX2+, SOX9+, and PDX1+; Figures 2A, 2C, and 2D) and gland cells (MUC6+, Figure 2B). Comparably, intestinal organoids demonstrate differentiation of intestinal-specific cell types such as goblet cells $\left(\mathrm{MUC2}^{+}\right.$, Figure 2E), enterocytes (KRT20 ${ }^{+}$, Figure 2F), enteroendocrine cells $\left(\mathrm{CHGA}^{+}\right.$, Figure $\left.2 \mathrm{G}\right)$, and proliferating cells $\left(\mathrm{Ki}^{+} 7^{+}\right.$, Figure $\left.2 \mathrm{H}\right)$.

\section{Discussion and Conclusions}

Organoids are culture models that recapitulate many of the properties of human tissues from which they are derived. For this reason, they have the potential to be a good alternative to the use of animal models. However, until recently, tissue availability for organoid generation constituted a major limitation that can be overcome by the use of hPSCs. We have demonstrated that both the STEMdiff' ${ }^{\text {TM }}$ Gastric Organoid and STEMdiff ${ }^{\mathrm{TM}}$ Intestinal Organoid Kits enable the generation of tissue-specific organoids from hPSCs in a highly reproducible manner. These workflows offer researchers an unlimited source of cells and robust procedures to generate organoid cultures and to conduct in vitro studies directly translatable to humans, thereby decreasing the reliance on traditional two dimensional cell line cultures and animal models.

\section{References}

1. McCracken KW, Catá EM, Crawford $\mathrm{CM}$, et al. Modelling human development and disease in pluripotent stemcell-derived gastric organoids. Nature 2014;516:400-4.

2. Spence JR, Mayhew CN, Rankin SA, et al. Directed differentiation of human pluripotent stem cells into intestinal tissue in vitro. Nature 2011;470:105-9.

3. Dedhia PH, Bertaux-Skeirik N, Zavros Y, Spence JR. Organoid Models of Human Gastrointestinal Development and Disease. Gastroenterology 2016;150:1098-112. 